\title{
An Integrated PHM Approach for Gas Turbine Engines
}

\author{
Alok R. Patnaik \\ Tecsis Corporation \\ 200-210 Colonnade Road, \\ Ottawa, ON K2E 7L5, Canada \\ Amiya Nayak \\ SITE, University of Ottwa, \\ 800 King Edward Ave., \\ Ottawa, ON K1N 6N5, Canada
}

\author{
V. Narasimhan \\ Faculty of Informatics and Communication \\ Central Queensland University - Melbourne Campus, \\ Australia \\ Prakash C. Patnaik \\ NRC - Institute for Aerospace Research, \\ M13 -1200 Montreal Road, \\ Ottawa, ON K1A 0R6, Canada
}

\begin{abstract}
The prognosis \& health management (PHM) of aerospace components is a very complex system. A complete PHM system involves sensors, signal processing, condition monitoring, health assessment, physical reasoning and decision- making. Such an integrated approach to prognostics involves physicsbased modelling, experience-based modelling and modelling based on the statistical properties of the components by using neural network and fuzzy logic techniques. In this paper, we give an overview of the PHM system currently being developed.
\end{abstract}

\section{Introduction}

Managing the health of any system is a challenging issue. The system under consideration must be monitored using sensors located at critical points. These sensors record physical parameters such as temperature, pressure, vibration. The first problem is to identify an anomaly among the data, either from a single sensor or from a group of sensors. The next problem is to be able to predict, using the current state of the system, the amount of time left before the system or the component fails, so that appropriate maintenance can be carried out. This is the basic idea of prognostic health management (PHM) system [1-4].

In general, an integrated PHM system consists of models of the system, set of sensors, facilities for data collection and analysis, and functions for detection of anomalies and then prediction of failures. Such an integrated system is designed to replace the scheduled maintenance of the system with a scheme where the system is maintained if and when necessary. Thus, the principle is to take corrective action only when and where it is needed.
Sensors play an essential part in PHM as these monitor the physical parameters of the system. Sensors, placed in strategic locations in the system, can be wired or wireless. Some of the sensors can be based on micro-electromechanical systems (MEMS). In particular, MEMS-based sensors are ideal for gas turbine engines.

In the context of aerospace industry, the life cycle management system has been largely based on experience [5]. With the advent of MEMS-based sensors, it opens up the possibility of monitoring the physical parameters at places where it was not possible to measure them, and therefore the state of system can be assessed. Any significant deviation from the expected behaviour of the system can be considered an anomaly and be dealt with appropriately and in a timely manner.

In this paper, we give an overview of such a PHM system under development for gas turbine engines. We describe various components of the PHM system, discuss different prognostics mechanisms, and present a high-level design of our PHM system.

\section{Components of a PHM}

\section{$\underline{\text { Sensors }}$}

Sensors are essential part of the PHM system. The issues here are the characteristics of the sensors such as the sensitivity of the detected signal, their location in the system, and whether these sensors should be wired or be wireless. Sensors are to be selected depending on the range of the parameter to be measured. For example, thin-film thermocouples, particularly made of $\mathrm{Pt} / \mathrm{Rh}$, are ideally suited to measure high temperatures on the surface of an engine blade. 
Model of the system

A mathematical or a geometrical description of the system is needed in order to model the location of the sensors. This is required to isolate the location of an anomaly. Often the sensor may not be located at site of a problem; however, if there is a grid of sensors, then it is possible to determine the site more precisely.

\section{Data Collection and Analysis}

Data from each sensor can be analysed to look for an anomaly. An anomaly can be defined as a datum which has a significant deviation from its characteristic behaviour. Depending on the requirement, several levels of alarms are set for each sensor. Since the sensors measure various physical parameters such as temperature, pressure, vibration, etc., the dataset will be multi-dimensional.

While each sensor is monitored individually for anomalies, it is often a challenge to identify a problem from the data (often noisy) from a set of sensors. The questions is: how early can we identify the problem so that we have more time to react and take preventive action. Concepts of the neural networks and Bayesian networks are used to locate a pattern in the multi-dimensional dataset.

Data from a single sensor are analysed to look for both the long term and the short term behaviour of the component. Any significant deviation is signalled as a possible problem. The severity level of the problem is determined as well as the time available to rectify it. Hence, appropriate action is taken.

\section{Approaches to Prognostics}

In the previous section, we mentioned the detection of an anomaly. The prediction that the anomaly can lead to a failure has three levels of analysis.

\section{Experience-based prognostics}

This is rather a simple and is common practice. One looks at the history of the failure of the system and any similarity with the previous failures suggest the appropriate action to be taken. Such experiencedbased decisions are rather ad hoc, often carried out without a proper understanding of the underlying problem. Its application is, therefore, limited.
Statistics-based Prognostics

In this approach, the collected data are analysed. Probability of failure are assigned to detected anomalies. The sensors measure various parameters inside an engine. These data are edited in an automated algorithm, and the statistical properties are determined continuously. Each data stream has its alert and alarm levels. At the basic level, trend curves are fitted continuously to the data to determine the current as well as the future behaviour. Alerts are usually set well before the measured parameters reach their respective critical levels, and appropriate corrective actions are taken. It is a challenge to do this in the harsh environment of the engines, as well as taking data from a large number of sensors. At the next level, the multidimensional dataset need to be searched for patterns which lead to failures. Any pattern recognition technique, namely neural networks or Bayesian belief networks, will be used in this context. Application of these techniques to the data from engines is a major advancement.

\section{Physics-based models}

The physics-based modelling involves solution of differential equations, often nonlinear, either in closed form or numerical approximations. The experience-based modelling involves empirical rules derived from failure history of the components. The probabilistic models such as Bayesian Belief Networks, Neural Networks or Fuzzy Systems can be used to predict the possible behaviour of the system using the current state of the system and the prior conditional probabilities, biases, and weights. Signals from various sensors are correlated to analyze and look for patterns of failures in the multidimensional data. We believe that the most effective means of prognostics in systems is the use of physics-based modelling and probabilistic models to develop decision making applications that can learn to locate these signatures of faults from existing data and can be used to predict possible failures

While the physics-based models are highly desirable, it is often difficult to build a physical model of a complex system. The physical and rulebased systems depend on the knowledge of the known fault events which are being monitored. The learning systems can process a wide variety of data types and perform superior to rule-based systems because they cover areas which are not covered by general rules. Therefore, the learning system works 
better where the physical models and general rules have not been developed. However, the learning systems are as good as the data on which they have been trained. An ideal system would be the one which can combine the benefits of all the three approaches.

In the context of gas turbine engines, the physicsbased models involve the study of lifetime of the engine blades, in particular, the lifetime of the thermal barrier coatings. The engine blades are made up of super-alloys based on Ni. These alloys are deformed at the operating temperature $\left(1200^{\circ} \mathrm{C}\right)$ of the engines and hence need thermal insulation. A bondcoat of $\mathrm{Ni}, \mathrm{Cr}, \mathrm{Al}, \mathrm{Y}$ is applied on the superalloy substrate and a coating of zirconia is grown on that which provides thermal insulation to the superalloy. Oxygen flows through zirconia and oxidises $\mathrm{Al}$ in the bondcoat. This oxidation process starts the diffusion of elements in the bondcoat. The thermal grown oxide layer is critical to the stability of the coating and hence the life of the engine blades. Several scenarios are being explored: internal oxidation, external oxidation of the super-alloy, and with and without the coating. The simulations help understand the growth of cracks in the oxide layers. Physical models will help predict the failures of the thermal barrier coating.

\section{PHM Architecture}

Figure 1 shows the high-level view of our PHM architecture. Due to page limitations, it is not possible to describe all the details of various components. We simply mention the major components of the system in this section.

Graphics Unit: This unit has the display functionalities such as line drawings, 3-D display, histograms, able to overlay curves of different sensors, a model of the system, location of the sensors, display multiple sensor data at the same time, interactively change the display etc.

Statistics Unit: It has the general statistical functions such as linear regression analysis, cubic spline fitting, short-term and long-term modelling of the data.

Data Mining and Data Fusion Unit: Since there are sensors of different kinds, the data streams from these sensors are to be analysed as a single entity. We use the concepts of data mining and fusion to accurately predict the health of the system.
Physics-based Model Unit: This unit implements the equations describing the thermal, electrical and other properties of the system. Some of them are differential equations which need to be solved numerically using finite element analysis. For example, the oxidation process in the engine blades, which impacts the lifetime, is described by a set of non-linear differential equations with moving boundary.

The biggest challenge for us has been in determining the location of the sensors and their physical characteristics. To facilitate this, we have a geometrical and a physical description of system so that we can identify the critical points that need to be monitored. The type of sensors depends on the level of signal to be detected. Since the sensors are based on MEMS technology, a large number of them can be easily installed without affecting the performance of the system while providing detail measurements. Obviously, this increases the load on data collection, storage and analysis. All these requirements are the inputs to our PHM system.

The data are processed and stored in the Data Storage. Each data stream (i.e. each sensor) is individually analysed using functions in the Statistics Unit. The Graphics Unit displays the raw as well as the processed data. Known patterns that have led to anomalies are searched in the multidimensional dataset in the Data Storage. The physical parameters measured are then fed to the mathematical equations describing the system, and the future state of the system is determined numerically.

The anomalies detected are then assessed for their severity and impact on the lifetime of the system. The risk factor associated with each anomaly is computed so that appropriate corrective action now can be taken.

\section{Conclusions}

In this paper, we have identified and described all components of an integrated PHM system where the sensors, data collections, modelling using statistical data analysis techniques, and numerical solutions to equations that describe the physical system can be incorporated in a natural fashion. We have also presented a high-level design of our PHM under development, keeping gas turbine engines in mind. Our approach is rather general as it can be easily adapted to any other system. 


\section{REFERENCES}

[1]C. S. Byington, M. Watson, M. J. Roemer, T. R. Galie, J. J. McGroarty and C. Savage. "Prognostic Enhancements to Gas Turbine Diagnostic Systems". Proc. IEEE Aerospace Conf., 2003

[2] A. Hess, L. Fila, "Prognostics, from the Need to Reality - from the Fleet Users and PHM System Designers/Developers Perspective", Proc. IEEE Aerospace Conf., 2002.

[3] G. J. Kacprzynski, M. J. Roemer, G. Modgil, A. Palladino, K. Maynard, "Enhancement of Physics-of-
Failure Prognostic Models with System Level Features, Proc. IEEE Aerospace Conf., 2004.

[4] S. Massam, S. McQuillan, "Verification of PHM Capabilities: a Joint Customer/Industrial Perspective", Proc. IEEE Aerospace Conf., 2002.

[5] X. Wu, J. Bird and P. C. Patnaik. "Advanced Prognosis for Life Cycle Management of Future and Legacy Aircraft propulsion Systems - a Canadian Perspective". Proc. 2nd Int. Symp. on Aerospace Materials and Manufacturing: Development, Testing, and Life Cycle Issues, 2004.

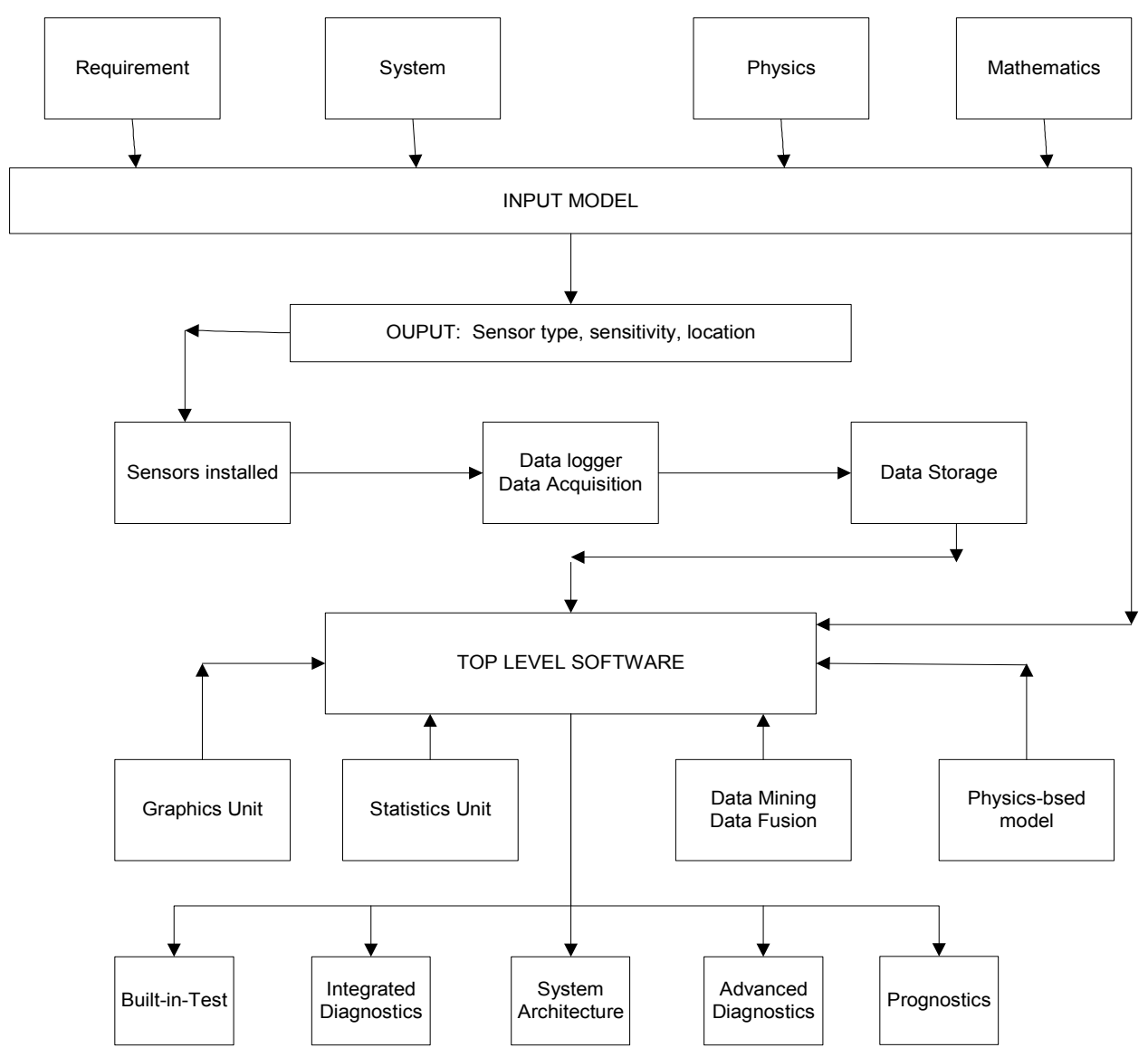

Figure 1: Block Diagram of our PHM Architecture 